\title{
Effects of tunneling on an IMPATT oscillator*
}

\author{
S. P. Kwok and G. I. Haddad \\ Electron Physics Laboratory, Department of Electrical Engineering. The University of Michigan, Ann Arbor, Michigan \\ 48104 \\ (Received 9 July 1971; in final form 21 October 1971
}

\begin{abstract}
A phenomenological formulation which incorporates both avalanche and tunneling mechanisms in an IMPATT diode is given. Here tunneling is viewed as a field-dependent carrier source. An electron after being field emitted may gain sufficient energy from the field to cause ionization. In this formulation, pure avalanche and pure tunneling appear as the two extreme cases of the general problem. The resultant general dc $I-V$ characteristic shows the dominance of tunneling at low voltages and the onset of the multiplication at higher voltages as observed experimentally. A small-signal admittance of an IMPATT oscillator with tunneling has been calculated. Under some conditions tunneling may increase the negative conductance. However as tunneling dominates, the negative conductance deteriorates and the oscillator will operate in the tunnel transit-time mode. Tunneling invariably shifts the frequency for optimum negative conductance upwards. The threshold frequency for negative conductance varies as the square root of current density for large multiplication factors as expected. However, for small ones it converges to a value determined only by the drift transit time. The general admittance expression reduces to that of pure avalanche and pure tunneling under the prescription $\omega_{a} \rightarrow \omega$ as $M \rightarrow \infty$ and $\omega_{a} \rightarrow \omega_{a z}$ as $M \rightarrow 1$, respectively. $\omega_{a}, \omega_{a 0}$, and $\omega_{a z}$ are the modified avalanche frequencies for the general case, pure avalanche, and pure tunneling, respectively.
\end{abstract}

\section{INTRODUCTION}

Due to the inherent transit-time nature of the IMPATT oscillator, a $p^{+} n n^{+}$diode designed to operate at a high frequency must have a narrow depletion width, a high internal electric field, and a low breakdown voltage. In a Si diode designed to operate above $300 \mathrm{GHz}$, the breakdown voltage is approximately $5 \mathrm{~V}$ or less and the internal field exceeds $10^{6} \mathrm{~V} / \mathrm{cm}$. At such a field strength tunneling becomes significant. ${ }^{1}$ Moreover, the inherent low-noise property of the tunneling process makes it potentially attractive to operate the IMPATT oscillator in the mixed tunnel and avalanche transit-time mode or tunnel-transit mode for low-noise applications. The theoretical feasibility of such a tunnel transit-time mode oscillation has been demonstrated ever since the inception of the Read diode ${ }^{2}$ and was later considered by others. ${ }^{3,4}$ Fabrication of a metal semiconductor structure for such operation was reported. However, tunnel transit-time mode oscillations have not yet been observed. 4

In this paper a phenomenological formulation which incorporates both tunneling and avalanche mechanisms is developed. The $I-V$ characteristic of a diode where the two mechanisms are present is calculated. The effects of tunneling on the small-signal admittance of an IMPATT oscillator is presented.

\section{TUNNELING IN A $p^{+} n$ ABRUPT JUNCTION}

The commonly used constant-field approximation of tunneling probability ${ }^{1,5-8}$ is inadequate for the calculation of tunneling current distribution throughout the junction which is essential for the formulation of the problem discussed later. Instead, a potential-barrier model indicated by the solid curve of Fig. 1 is used for the calculation of direct tunneling probability of electrons having energy 8 . The right-hand portion of the potential-barrier energy is determined by the uniform impurity space charge. Taking the energy reference as shown in Fig. 1, the right-hand portion of the potential barrier energy is given by

$$
U(x)=\left(q^{2} N_{D} / 2 \epsilon\right)(x-w)^{2}-q\left(V_{b}+V_{a}\right),
$$

where the width $w$ is given by

$$
w=\left[\left(2 \epsilon / q N_{D}\right)\left(V_{b}+V_{a}\right)\right]^{1 / 2} \text {. }
$$

The left-hand portion is given by the vertical line. The corresponding field profile is triangular with its maximum at $x=0$. The tunneling probability is then calculated by the WKB approximation to be ${ }^{9}$

$$
\begin{aligned}
D\left(\mathcal{E}, \mathscr{E}_{1}\right)= & P^{M} \exp \left(-\left\{\left(4 m^{*} \epsilon / q^{2} N_{D} \hbar^{2}\right)\right.\right. \\
& \left.\left.\times\left[q\left(V_{b}+V_{a}\right)+\mathcal{E}_{g}-|\mathcal{E}|-\mathcal{E}_{\perp}\right] \mathcal{E}_{\mathscr{E}}\right\}^{1 / 2}\right),
\end{aligned}
$$

where

$$
\begin{aligned}
& P \equiv \frac{\left[q\left(V_{b}+V_{G}\right)+\mathcal{E}_{g}-|\mathcal{E}|-\mathcal{E}_{\perp}\right]^{1 / 2}+\left(\mathscr{E}_{g}\right)^{1 / 2}}{\left[q\left(V_{b}+V_{a}\right)-|\mathscr{E}|-\mathcal{E}_{\perp}\right]^{1 / 2}}, \\
& M \equiv\left(\frac{4 m^{*} \epsilon}{q^{2} N_{D}}\right)^{1 / 2} \frac{\left[q\left(V_{b}+V_{a}\right)-|\mathcal{E}|-\mathcal{E}_{\perp}\right]}{\hbar}, \\
& V_{b}=\text { the built -in barrier potential, } \\
& V_{a}=\text { the applied voltage, } \\
& \mathcal{E}=\text { the total energy } \equiv \mathscr{E}(x), \\
& \mathcal{E}_{\perp}=\text { the kinetic energy associated with transverse } \\
& \quad \text { momentum, } \\
& m^{*}=\text { the reduced effective mass, } \\
& \mathcal{E}_{g}=\text { the band-gap energy, } \\
& q=\text { the electronic charge, } \\
& N_{D}=\text { the donor impurity density, } \\
& \epsilon=\text { the dielectric constant of the semiconductor, and }
\end{aligned}
$$




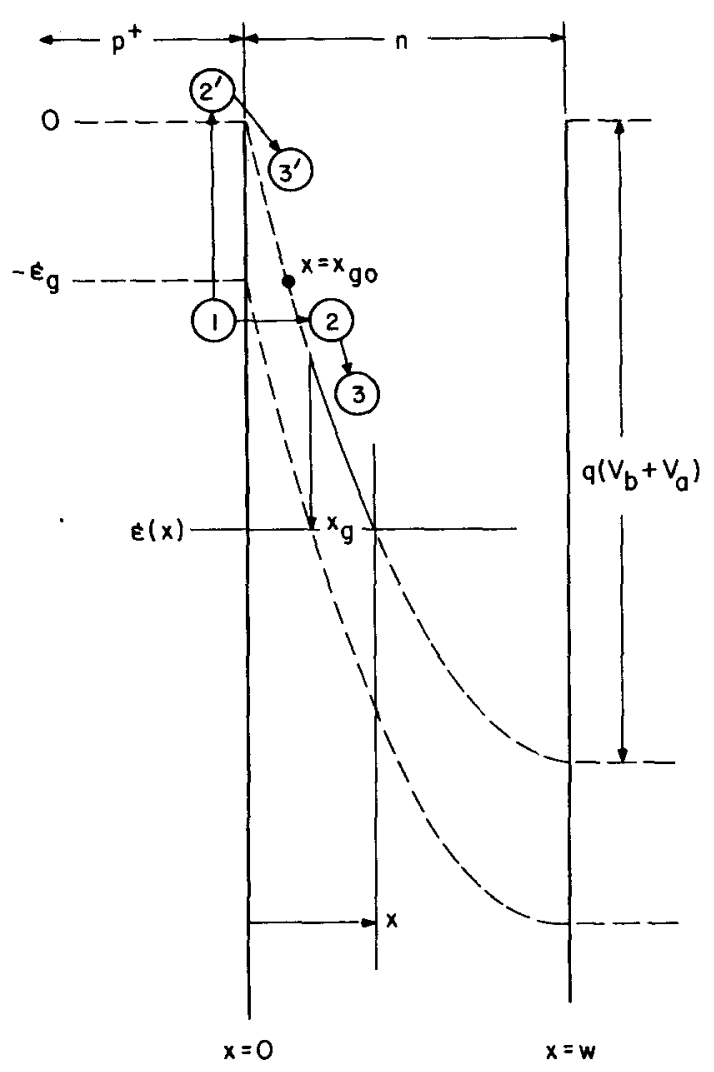

FIG. 1. Band structure of a $p^{+} n$ junction. 1-2 electron tunneling; $2-3$ electron acceleration. $1-2^{\prime}$ thermionic emission; $2^{\prime}-3$ ' electron diffusion and acceleration.

\section{$\hbar=$ Planck's constant divided by $2 \pi$.}

A priori, tunneling probability is significant only near $x=0$ where the field is highest. Since in direct tunneling the energy levels of the initial and the final states are conserved, association of energy level with the position $x$ where a tunneling electron appears at the conduction band edge, leads to the conclusion that mainly electrons with energy levels near the top of the valence band contribute to the tunneling current. Moreover, as can be seen in Fig. 2, the higher the applied bias voltage, the smaller is the region where most of the tunneling cur rent is confined. For the case of $q\left(V_{b}+V_{a}\right) \gg \mathcal{E}_{g}+\mathscr{E}_{\perp}$ $+|\mathcal{E}|$, the expression simplifies approximately to ${ }^{9}$

$$
D\left(\mathcal{E}, \mathcal{E}_{1}\right)=\exp \left[-\left(\sqrt{2} m^{* 1 / 2} \mathcal{E}_{g}^{3 / 2} / q \hbar E_{m}\right)\right]
$$

which has the general form of the constant-field tunneling probability. The general form of the constantfield tunneling probability is

$$
D=D_{0} \exp \left[-C_{0}\left(m^{*^{1 / 2}} \xi_{g}^{3 / 2} / q \hbar E\right)\right],
$$

where $C_{0}$ and $D_{0}$ are coefficients of order unity whose values depend on the model or the approach used. ${ }^{6-8}$ Such agreement is consistent with the fact that when the applied voltage is large the field is effectively constant in the small region of current confinement. However, the values of voltages of interest are relatively small so that the expression of Eq. (3) will be used for calculation of tunneling current. The net tunneling current density $J_{\varepsilon}$ is given by the general expression ${ }^{10}$

$$
J_{z}=\left(q / 2 \pi^{2} h\right) \int_{-\infty}^{\infty} d \mathscr{E}\left[f(\mathcal{E})-f\left(\mathcal{E}+q V_{a}\right)\right] \int d^{2} k_{t} D\left(\mathcal{E}, \mathcal{E}_{1}\right),
$$

where $k_{t}$ is the transverse wave vector and $f(\mathscr{E})$ is the Fermi-Dirac distribution function. The subscript $z$ pertains to Zener (tunneling) current. Conservation of transverse momentum in direct tunneling restricts the domain of integration of $k_{t}$ to

$$
\begin{aligned}
& 0 \leqq k_{t}^{2} \leqq \text { Minimum }\left\{\left(2 m_{p}^{*} / \hbar^{2}\right)\left(|\mathcal{E}|-\mathcal{E}_{g}\right),\right. \\
& \left.\left(2 m_{n}^{*} / \hbar^{2}\right)\left[q\left(V_{b}+V_{a}\right)-|\mathcal{E}|\right]\right\},
\end{aligned}
$$

where the subscripts $p$ and $n$ pertain to the $p$ - and $n$ regions, respectively. If the proper limits of integration are inserted, the tunneling current density, Eq. (6), becomes

$$
\begin{aligned}
J_{\mathscr{z}}= & \left(4 \pi q m^{*} / \hbar^{3}\right)\left\{\int_{-\mathcal{E}_{g}}^{-\delta_{i}} d \mathcal{E}\left[f(\mathcal{E})-f\left(\mathcal{E}+q V_{a}\right)\right]\right. \\
& \times \int_{0}^{\left|\mathcal{E}_{1}\right| \mathcal{\delta}_{\mathcal{E}}} d \mathcal{E}_{\perp} D\left(\mathcal{E}, \mathcal{E}_{\perp}\right) \\
& +\int_{-\delta_{i}}^{-q_{(}\left(V_{b}+V_{a}\right)} d \mathcal{E}\left[f(\mathcal{E})-f\left(\mathcal{E}+q V_{a}\right)\right] \\
& \left.\times \int_{0}^{a\left(V_{b}+V_{a}\right)-|\mathcal{E}|} d \mathcal{E}_{\perp} D\left(\mathcal{E}, \mathcal{E}_{\perp}\right)\right\}
\end{aligned}
$$

where

$$
\mathcal{E}_{i} \equiv \frac{m_{n}^{*} q\left(V_{b}+V_{a}\right)+m_{p}^{*} \mathcal{E}_{g}}{m_{n}^{*}+m_{p}^{*}} .
$$

The calculated tunneling current densities obtained from Eqs. (3) and (7) for abrupt GaAs junctions are plotted as the dashed lines in Fig. 3.

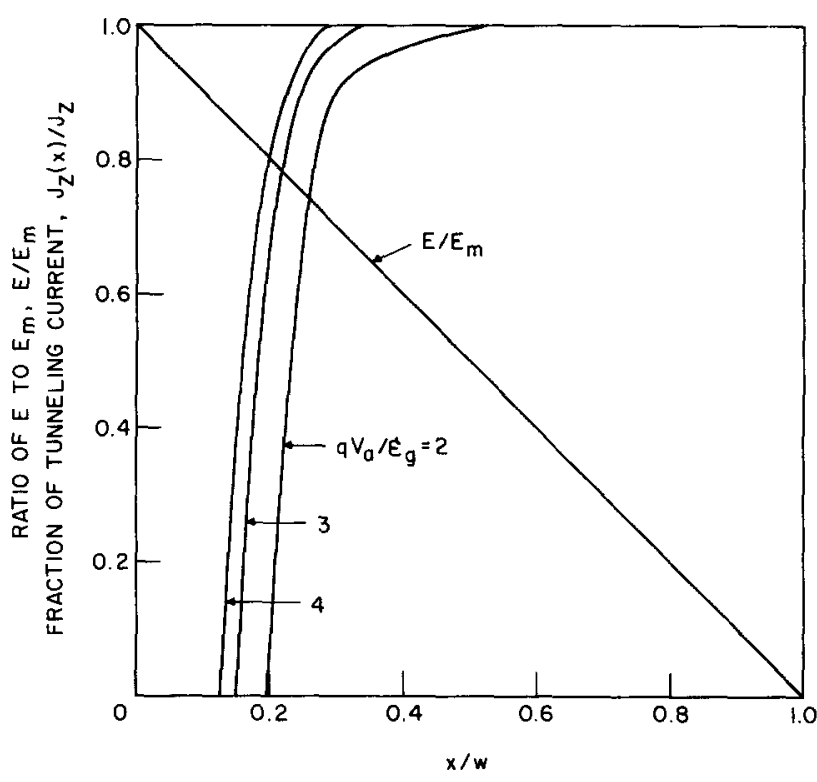

FIG. 2. Current distribution and field profile. GaAs $p^{+} n$ (Abrupt), $N_{A}=10^{20} / \mathrm{cm}^{3}, N_{D}=10^{18} / \mathrm{cm}^{3}, \mathcal{E}_{B}=1.43 \mathrm{eV}, m^{*}=0.06$, and $T=300^{\circ} \mathrm{K}$ 


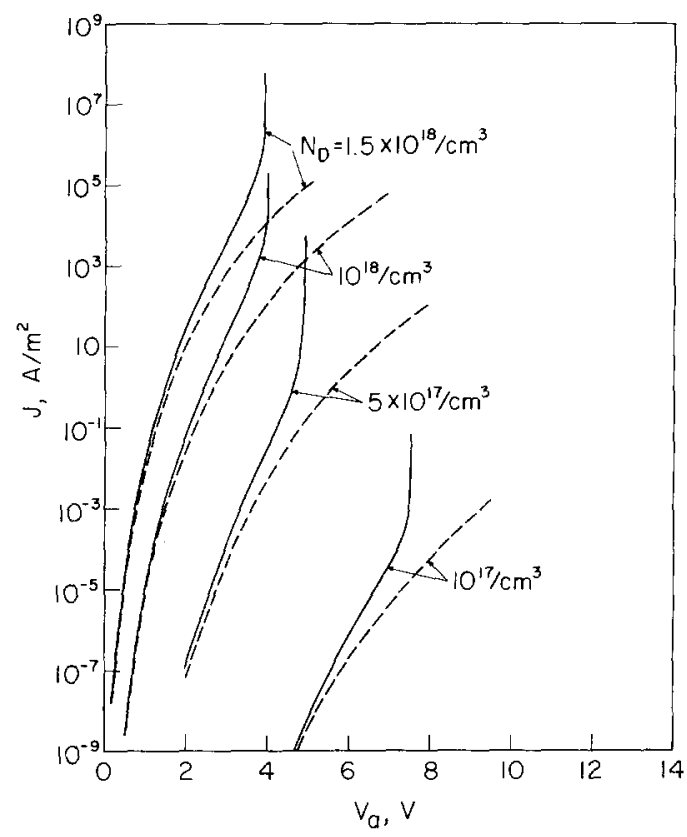

FIG. 3. Theoretical $I-V$ characteristics of an abrupt junction with tunneling and multiplication. $-J_{0}$, total current density; $--J_{z}$, tunneling current density; GaAs $p^{+} n$ (Abrupt), $\varepsilon_{g}=1.43$ $\mathrm{eV}, \epsilon=10.9, m^{*}=0.06, T=300^{\circ} \mathrm{K}, N_{A}=10^{20} / \mathrm{cm}^{3}, J_{0}=J_{z} /$ $\left(1-\int_{0}^{\omega} \alpha d x\right), \alpha=A \exp (-b / E)^{m}, m=2, A=3.5 \times 10^{5} / \mathrm{cm}$, $b=6.85 \times 10^{5} \mathrm{~V} / \mathrm{cm}$.

\section{TUNNELING CURRENT DISTRIBUTION}

For the purpose which will become apparent later, the tunneling current density distribution $J_{g}(x)$ is defined as the magnitude of the tunneling current density due to electrons whose energy range is $[0, \mathcal{E}(x)]$, as shown in Fig. 1. Clearly,

$$
\begin{aligned}
& J_{z}(x)=J_{z}\left(x_{\mathrm{g} 0}\right)=0 \text { for } x \leqq x_{\mathrm{g} 0}, \\
& J_{z}(x)=J_{z}(w)=J_{z} \text { for } x \geqq w, \\
& \frac{\partial J_{z}(x)}{\partial x} \geqq 0 .
\end{aligned}
$$

The total tunneling current density $J_{z}$ is different from the current density distribution $J_{z}(x)$ whose value at $x=w$ is equal to the former. In a direct tunneling process, conservation of energy leads directly to the as sociation of an electron energy level $\mathscr{E}(x)$ with the position $x$ where it appears at the conduction band edge. This association is done by equating the energy $\mathcal{E}$ with $U(x)$ of Eq. (1) which yields

$$
x=\left(\frac{2 \epsilon}{q N_{D}}\left(V_{b}+V_{a}\right)\right)^{1 / 2}\left[1-\left(1-\frac{|\mathcal{E}|}{q\left(V_{b}+V_{a}\right)}\right)^{1 / 2}\right] .
$$

The tunneling current distributions for a $p^{+} n$ GaAs diode calculated from Eqs. (3), (7), and (9) are given in Fig. 2. Due to the band gap, there is no tunneling current in the immediate vicinity of the $p^{+}$side. However, the region where tunneling current emanates and reaches $90 \%$ of its total invariably spans only about $10 \%$ of the total depletion layer.
In an indirect band-gap material, tunneling requires the cooperation of a phonon $(s)$ in order to absorb the momentum imbalance of the initial and final states. How ever, the qualitative feature of the direct tunneling current is retained. The phonon fine structures can be neglected, but, the magnitude is reduced due to the reduction of the tunneling probability. ${ }^{8}$ The direct association of the energy and position of an electron in the tunneling current distribution is clouded by such phonon cooperations. However, since the phonon energies ( $\leqslant 58 \mathrm{meV}$ in $\mathrm{Si}$ ) are invariably much less than the bias potential, which is of the order of several band gaps, the resultant energy-position uncertainty becomes negligible. Admittedly, a serious problem may arise from multiplephonon-assisted tunneling, but such an event has a very small probability of occurrence and hence can be neglected here.

\section{PHENOMENOLOGICAL FORMULATION OF TRANSPORT EOUATIONS INVOLVING AVALANCHE AND TUNNELING PROCESSES}

The observation of the dominance of tunneling current and the set in of multiplication at a higher voltage in the $I-V$ characteristics of a narrow $p-n$ junction led Chynoweth and McKay ${ }^{1}$ to suggest that an electron, after being field emitted (tunneling), may acquire sufficient energy from the field to cause ionization. In the same manner the following assumptions are made:

(a) The tunneling current arises from the net rate of electrons which tunnel from the valence band to the conduction band edges. For each electron leaving the valence band edge a hole is created there.

(b) Both an electron and a hole thus created drift under the influence of the field in their respective bands, just as other carriers present there by other means.

(c) If the field is high enough, the carriers may gain enough energy to produce electron-hole pairs in the usual manner of avalanche multiplication.

(d) For convenience the diffusion current will be neglected here.

Mathematically, the following is obtained:

$$
\begin{aligned}
& J_{n}=-q n v_{n}, \\
& J_{p}=-q p v_{p}, \\
& \frac{\partial n}{\partial t}=\frac{1}{q} \frac{\partial J_{n}(x)}{\partial x}+\alpha_{n} v_{n} n+\alpha_{p} v_{p} p+\frac{1}{q} \frac{\partial J_{g}(x)}{\partial x}, \\
& \frac{\partial p}{\partial t}=-\frac{1}{q} \frac{\partial J_{p}(x)}{\partial x}+\alpha_{n} v_{n} n+\alpha_{p} v_{p} p+\frac{1}{q} \frac{\partial J_{s}\left(x+x_{s}\right)}{\partial x}, \\
& \frac{\partial E}{\partial x}=\frac{q}{\epsilon}\left(N_{D}+p-n\right),
\end{aligned}
$$

and

$$
\nabla \cdot \vec{J}_{t} \equiv \nabla \cdot\left(\vec{J}_{c}+\epsilon \frac{\partial \vec{E}}{\partial t}\right)=0
$$

where

$$
\vec{J}_{c} \equiv \vec{J}_{n}+\vec{J}_{p}
$$


The subscripts $n$ and $p$ pertain to electrons and holes, respectively; $\alpha$ is the ionization rate; $v$ is the saturated velocity; $n$ and $p$ are the electron and hole densities, respectively, and $x_{\mathrm{g}}$ is the spatial separation of the valence band and the conduction band edges at the same energy. The polarity of the current densities is defined to be negative when flowing in the positive $x$ direction under reverse bias. $J_{2}(x)$, being tunneling current density distribution, is positive by definition. The term $\partial J_{z}\left(x+x_{g}\right) / \partial x$ appearing in the hole continuity equation is due to the fact that the rate of increase of the hole population at $x$ due to tunneling is equal to that of electrons at $x+x_{g}(x)$ as shown in Fig. 1. In writing $\partial J_{g}\left(x+x_{g}\right) / \partial x$, it is assumed that a tunneling electron appears at the conduction band edge simultaneously with the occurrence of a hole in the valence band. This assumption is valid provided the period of any excitation is long compared to the tunneling time of flight to be discussed later. Let $J_{n s}, J_{p s}$, and $J_{s}$ be the magnitude of electron and hole saturation-current densities and their sum, respectively. For convenience, assume $\alpha_{n}=\alpha_{p}=\alpha$. Equations $(10)-(15)$ can be manipulated with the boundary conditions:

$$
\begin{aligned}
& J_{p}(0, t)=J_{c}(0, t)+J_{n s}, \quad J_{p}(w, t)=-J_{p s}, \\
& J_{n}(0, t)=-J_{n s}, \quad J_{n}(w, t)=J_{c}(w, t)+J_{p s}, \\
& J_{\varepsilon}(0, t)=0, \quad J_{\varepsilon}(w, t)=J_{\varepsilon}(t)
\end{aligned}
$$

to yield

$$
\frac{d}{d t} J_{t}(t)+A(t) J_{t}(t)=Y(t)
$$

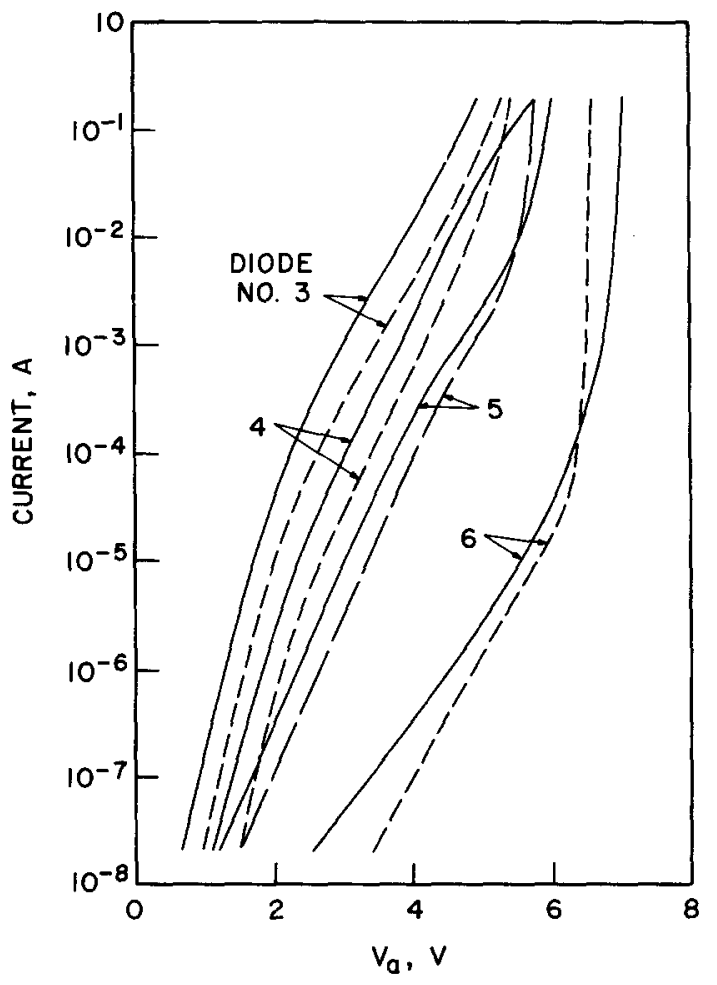

FIG. 4. Measured $I-V$ characteristics of abrupt junctions with tunneling and multiplication. (Singh Tyagi, Ref. 12), Si $p^{*} n$ (Abrupt) $-80^{\circ} \mathrm{C},---76^{\circ} \mathrm{C}--0^{\circ} \mathrm{C}$. where

$$
A(t) \equiv\left[\left(v_{n}+v_{p}\right) / w\right]\left[1-\int_{0}^{w} \alpha(x, t) d x\right]
$$

and

$$
\begin{aligned}
Y(t) \equiv & -\frac{v_{n}+v_{p}}{w}\left[J_{s}+J_{z}(t)\right]+\frac{\epsilon}{w}\left(\frac{d^{2}}{d t^{2}} \int_{0}^{w} E(x, t) d x-\left(v_{n}+v_{p}\right)\right. \\
& \left.\times \int_{0}^{w} \alpha(x, t) \frac{\partial E(x, t)}{\partial t} d x+v_{p} \frac{d E(0, t)}{d t}+v_{n} \frac{d E(w, t)}{d t}\right) .
\end{aligned}
$$

The proper initial value for a periodic solution is ${ }^{11}$

$$
J_{t}(0)=\int_{0}^{T} Y(t) \exp \left[\int_{0}^{t} A\left(t^{\prime}\right) d t^{\prime}\right] d t\left\{\exp \left[\int_{0}^{T} A(t) d t\right]-1\right\}^{-1},
$$

where $T$ is the period.

\section{dc CHARACTERISTICS}

Setting all terms involving the time derivative equal to zero, the dc current density is given by

$$
J_{c}=-\frac{J_{s}+J_{z}}{1-\int_{0}^{w} \alpha d x} .
$$

The tunneling current appears as a field-dependent saturation current. At low voltages when the ionization integral is negligible, tunneling dominates. As the bias voltage is increased, multiplication sets in. These features are clearly indicated in the plots of Eq. (18) in Fig. 3 and the measured characteristics ${ }^{12}$ given in Fig. 4. In Fig. 3 the saturation-current density has been assumed to be negligible compared to that of tunneling. Due to the lack of data, the ionization integrals were evaluated using the value of ionization rates due to Sze and Gibbons, ${ }^{13}$ although at very high fields $\left(\gtrsim 10^{6}\right.$ $\mathrm{V} / \mathrm{cm}$ ) such values are expected to be different. Clearly, the qualitative agreement between theory and measure ment is very good.

\section{DYNAMIC CASES}

According to the earlier discussion, the tunneling current is concentrated in a small region where the field is highest. It is also known that avalanche multiplication behaves similarly. It can be assumed, for mathematical expediency, that all tunneling and ionization are confined to a small region $[0, \delta]$ where the field is high and assumed to be constant. Outside $[0, \delta]$ the field takes up a considerably lower value but is still high enough to give rise to saturated drift velocities. Using the property of Eq. (15), Eq. (16) readily simplifies to

$$
\frac{\delta}{v_{n}+v_{p}} \frac{d J_{c}}{d t}+\left(1-\int_{0}^{\delta} \alpha d x\right) J_{c}=-\left(J_{s}+J_{\ell}\right)
$$

For the pure avalanche case it becomes the familiar expression

$$
\frac{\delta}{v_{n}+v_{p}} \frac{d J_{c}}{d t}+\left(1-\int_{0}^{\delta} \alpha d x\right) J_{c}=-J_{s},
$$

and for the pure tunneling one,

$$
\frac{\delta}{v_{n}+v_{p}} \frac{d J_{c}}{d t}+J_{c}=-\left(J_{s}+J_{s}\right)
$$


The latter is the current equation of the tunnel transittime oscillator which was considered by other workers. ${ }^{2-4}$ Under the constant-field assumption, the direct tunneling current expression due to $\mathrm{Kane}^{7}$ at zero temperature is given by

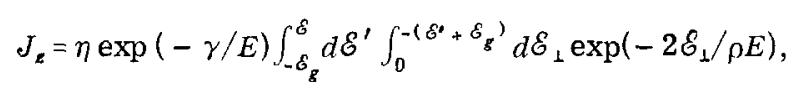

where

$$
\begin{aligned}
& \eta=q m^{*} / 18 \hbar^{3}, \\
& \gamma=\pi m^{* 1 / 2} \mathcal{E}_{g}^{3 / 2} / 2 \sqrt{2} \hbar q,
\end{aligned}
$$

and

$$
\rho=\sqrt{2} \hbar q / 2 \pi m^{*_{1 / 2}} \mathcal{E}_{g}^{1 / 2} .
$$

The domain of integration of $\mathcal{E}_{1}$ is taken to be $\leqq|\mathcal{E}|-\mathcal{E}_{\mathcal{g}}$ Using the relation $\mathscr{E}=-q E x$, the current density distribution is obtained:

$$
\begin{aligned}
J_{\boldsymbol{g}}(x)= & \eta \rho E \exp (-\gamma / E)\left(q E x-\mathcal{E}_{\boldsymbol{g}}-\rho E\right. \\
& \left.\times\left\{1-\exp \left[\left(-q E x+\mathscr{E}_{\boldsymbol{g}}\right) / \rho E\right]\right\}\right) \text { for } \delta \geqq_{x}>\mathcal{E}_{\boldsymbol{g}} / q E \\
= & 0 \quad \text { for } x \leqq \mathcal{E}_{g} / q E .
\end{aligned}
$$

The tunneling current density is simply $J_{z}(\delta)$. Substituting typical values $E \sim 10^{6} \mathrm{~V} / \mathrm{cm}, \mathcal{E}_{\mathrm{g}} \sim 1 \mathrm{eV}, \gamma / E$ $\sim 20$, and using

$$
q \delta E-\mathcal{E}_{g} \gg \rho E,
$$

the derivative of the tunneling current density can be approximated by

$$
J_{x}^{\prime}(\delta)=\frac{\partial J_{z}(\delta)}{\partial E}=J_{z}(\delta) \frac{\gamma / E+2}{E} .
$$

Using a small-signal procedure similar to that of Gilden and Hines, ${ }^{14}$ the following quantities are defined:

$$
\begin{aligned}
& J_{c}=J_{c 0}+\tilde{j}_{c} e^{i \omega t}, \\
& \alpha=\alpha_{0}+\alpha_{0}^{\prime} \tilde{E} e^{i \omega t}, \\
& J_{z}(\delta)=J_{z 0}(\delta)+J_{z 0}^{\prime}(\delta) \tilde{E} e^{i \omega t}
\end{aligned}
$$

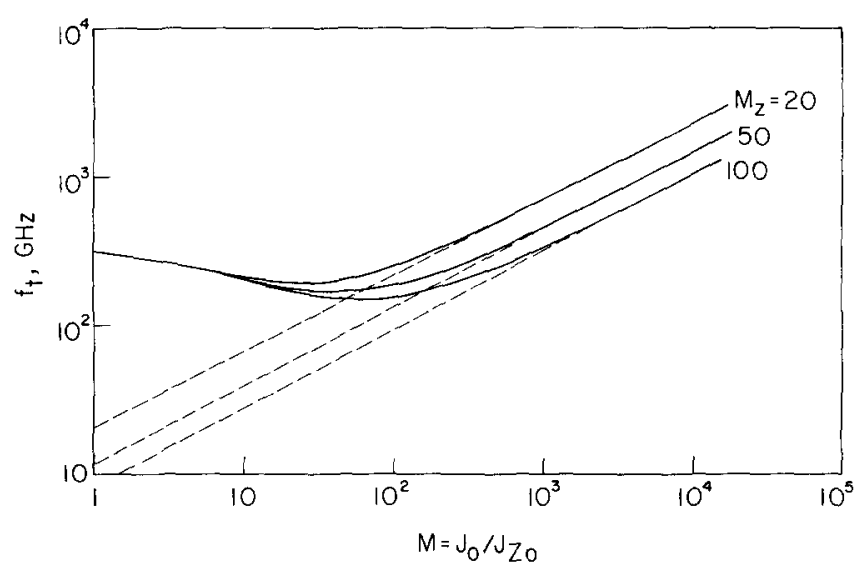

FIG. 5. Threshold frequency vs multiplication factor. $f_{a z}$ $=100 \mathrm{GHz}, \tau_{a}=0.1 / 2 \pi f_{a z}, \tau=10 \tau_{a}, f_{a z}=\omega_{a z} / 2 \pi$.

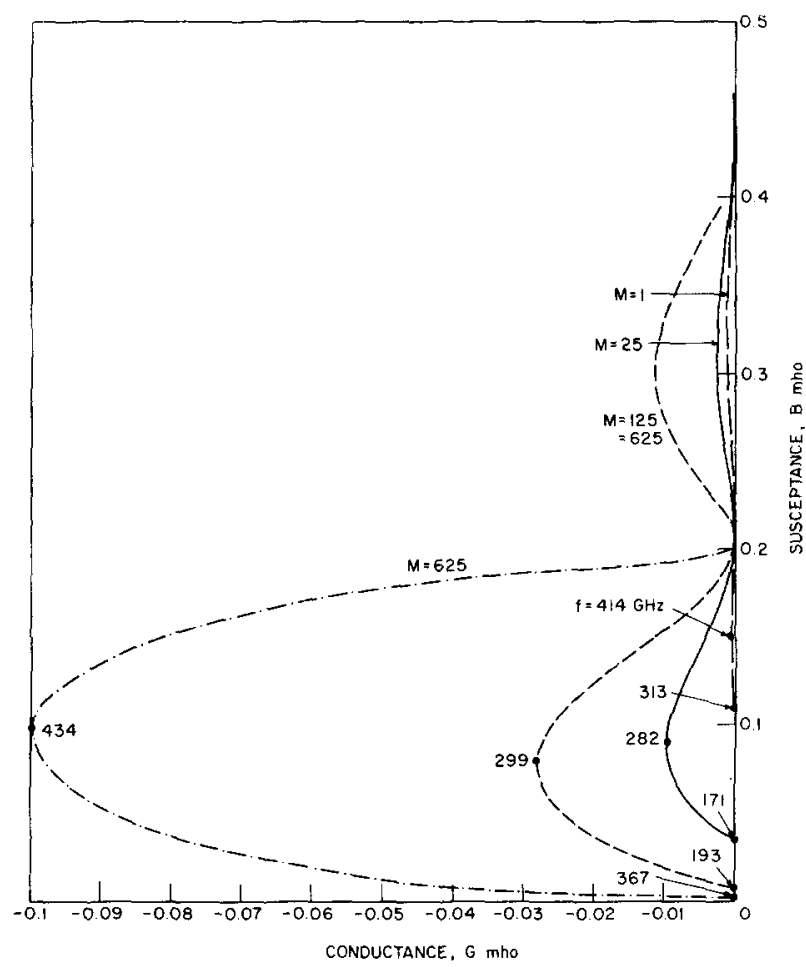

FIG. 6. Small-signal admittance of an IMPATT oscillator.

The subscript 0 pertains to the dc operating point and $\tilde{j}_{c}$ and $\tilde{E}$ are the small-signal varying amplitudes. The prime indicates the derivative with respect to electric field. Following this procedure, the impedances of the avalanche region and the drift region are given, respectively, by

$$
\begin{aligned}
Z_{a}= & \frac{(2 / M)^{2}+\left(\omega \tau_{a}\right)^{2}}{\omega c_{a}\left(\left\{(2 / M)^{2}+\omega^{2} \tau_{a}^{2}\left[1-\left(\omega_{a} / \omega\right)^{2}\right]\right\}^{2}+\left[\left(2 \omega_{a} / \omega\right)\left(\omega_{a} \tau_{a} / M\right)\right]^{2}\right)} \\
& \times\left\{\left(\frac{2 \omega_{a}}{\omega} \frac{\omega_{a} \tau_{a}}{M}\right)-i\left[\left(\frac{2}{M}\right)^{2}+\omega^{2} \tau_{a}^{2}\left(1-\left(\omega_{a} / \omega\right)^{2}\right)\right]\right\}
\end{aligned}
$$

and

$$
\begin{aligned}
Z_{d}= & \left\{\omega c_{d}\left[\left(1-\frac{\omega^{2}}{\omega_{a}^{2}}\right)^{2}+\left(\frac{2 \omega}{\omega_{a}} \frac{1}{\omega_{a} \tau_{a} M}\right)^{2}\right]\right\}^{-1}\left\{\left[\frac{\sin \theta}{\theta} \frac{\omega}{\omega_{a}} \frac{2}{\omega_{a} \tau_{a} M}\right.\right. \\
& \left.+\frac{1-\cos \theta}{\theta}\left(1-\frac{\omega^{2}}{\omega_{a}^{2}}\right)\right]+i\left[\frac{\sin \theta}{\theta}\left(1-\frac{\omega^{2}}{\omega_{a}^{2}}\right)-\left(\frac{2 \omega}{\omega_{a}} \frac{1}{\omega_{a} \tau_{a} M}\right)^{2}\right. \\
& \left.\left.-\left(1-\frac{\omega^{2}}{\omega_{a}^{2}}\right)^{2}-\frac{1-\cos \theta}{\theta} \frac{\omega}{\omega_{a}} \frac{2}{\omega_{a} \tau_{a} M}\right]\right\},
\end{aligned}
$$

where $\omega$ is the radian frequency of operation, $\theta$ is the drift region transit angle, $c_{a}$ and $c_{d}$ are the capacitances of the avalanche and drift regions, respectively, and $\tau_{a}$ is equal to $2 \delta /\left(v_{n}+v_{p}\right)$. The modified "avalanche" frequency $\omega_{a}$ is defined as

$$
\omega_{a}=\left\{\left(2 / \tau_{a} \epsilon\right)\left[\alpha_{0}^{\prime} \delta J_{c 0}+J_{k 0}^{\prime}(\delta)\right]\right\}^{1 / 2} .
$$

For the case of pure avalanche it becomes the conventional expression ${ }^{14}$

$$
\omega_{a 0}=\left(2 \alpha_{0}^{\prime} \delta_{d} J_{c 0} / \tau_{a} \epsilon\right)^{1 / 2} .
$$




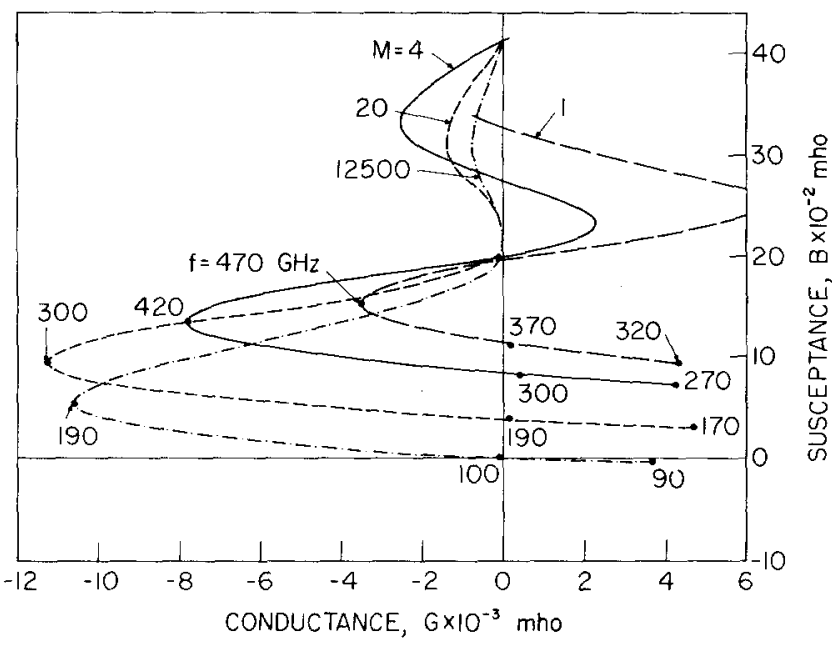

FIG. 7. Small-signal admittance of an IMPATT oscillator for constant $\alpha^{\prime} . \quad J=\left(2 \pi f_{a 0}\right)^{2} /\left(2 \alpha^{\prime} \delta / \tau_{a} \epsilon\right), f_{a 0}=100 \mathrm{GHz}, \omega_{a}=\omega_{a 0} \times$ $(1+20 / M)^{1 / 2}, \omega_{a 0} \tau_{a}=0.1, A=10^{-10} m^{2}, \delta / W=0.1$, and $\epsilon_{r}=10.9$.

On the other extreme of pure tunneling it simplifies to

$$
\omega_{a \varepsilon}=\left[\left(2 / \tau_{a} \epsilon\right) J_{\varepsilon 0}^{\prime}(\delta)\right]^{1 / 2} \text {. }
$$

The total diode impedance is

$$
Z=Z_{a}+Z_{d}+R_{s}
$$

where $R_{s}$ is the residual resistance, which in the discus sion here will be assumed to be negligible. The admittance is simply the inverse of $Z$. The impedances (admittances) for pure avalanche and pure tunneling can be obtained from the general one by setting $\omega_{a} \rightarrow \omega_{a 0}$ as $M-\infty$ and $\omega_{a}-\omega_{a \varepsilon}$ as $M-1$, respectively, where $M$ is the dc multiplication factor $\left(1-\int_{0}^{6} \alpha_{0} \mathrm{dx}\right)^{-1}$. Such a procedure is equivalent to letting, respectively, $J_{z}(\delta)=0$ and $\int_{0}^{\delta} \alpha d x=0$ in Eq. (19) prior to making the linearization.

There are two ways of investigating the effects of tunneling on the performances of an IMPATT oscillator:

(i) Bias the diode near its breakdown voltage and vary the multiplication factor and hence the dc current density while effectively keeping the other parameters $\alpha_{0}^{\prime}, \delta, E$, and $J_{z 0}(\delta)$ constant. The modified avalanche frequency can be shown from Eqs. (23), (27), (29), and $J_{c 0} \simeq M J_{z 0}$ to vary with $M$ as

$$
\omega_{a}=\omega_{a \varepsilon}\left(1+M / M_{\varepsilon}\right)^{1 / 2},
$$

where

$$
M_{\varepsilon} \equiv \frac{\gamma / E_{0}+2}{\alpha_{0}^{\prime} E_{0} \delta} \sim 50
$$

From the admittance calculation it is found that the threshold frequency (the frequency below which there is no negative conductance) varies as the usual square root of the dc current density for large values of $M$ such that $M \gg M_{z}$. However, as the value of $M$ is reduced and eventually approaches unity, the threshold frequency instead of decreasing as the square root of the current density, converges to one which is determined only by the transit time in the drift region as shown in
Fig. 5. The admittance of the oscillator given in Fig. 6 indicates a decrease in the negative conductance with a decreasing value of $M$ due to the overwhelming influence of the current density.

(ii) To avoid the overshadowing effects of current density consider hypothetical diodes having the same crosssectional area, the same active and drift region widths, and are biased at the same total dc current. The effects of tunneling will be viewed by varying the value of $M$.

Assume $\alpha_{0}^{\prime}$ to be constant. Here, $\omega_{a}$ is most conveniently expressed as

$$
\omega_{a}=\omega_{a 0}\left(1+M_{z} / M\right)^{1 / 2}
$$

The admittances are plotted for different values of $M$. It is found that the negative conductance in fact is larger for $M \sim M_{z}$, as is the frequency, than for the pure avalanche case $M \gg M_{z}$. However, it diminishes as $M$ approaches unity. It is suggested that such behavior is physically consistent. The tunneling current serves as a field-dependent source of carriers. Though the current has a zero phase lag relative to the voltage, the injected carriers may either drift without ionization or undergo ionization gaining the usual $90^{\circ}$ phase lag. If multiplication is prevalent it is possible to increase the negative conductance at a higher frequency. However, if tunneling is dominant the field-emitted carriers will have little chance of being multiplied, thus the zero-phase nature of the tunneling carriers becomes dominant the reby reducing the negative conductance, as shown in Fig. 7. As expected both the threshold frequency and the optimum frequency of operation shift upward as tunneling is enhanced. It is still inconclusive, however, to construe the slight increase of the negative conductance due to tunneling as an increase in power and/or efficiency. For a constant total dc current the drop in value of large $M$ to $\sim M_{z}$ necessarily means that the diode has a soft breakdown characteristic. A large-signal analysis may indicate a significant drop in power and/or efficiency. Another reason is discussed in the following paragraph.

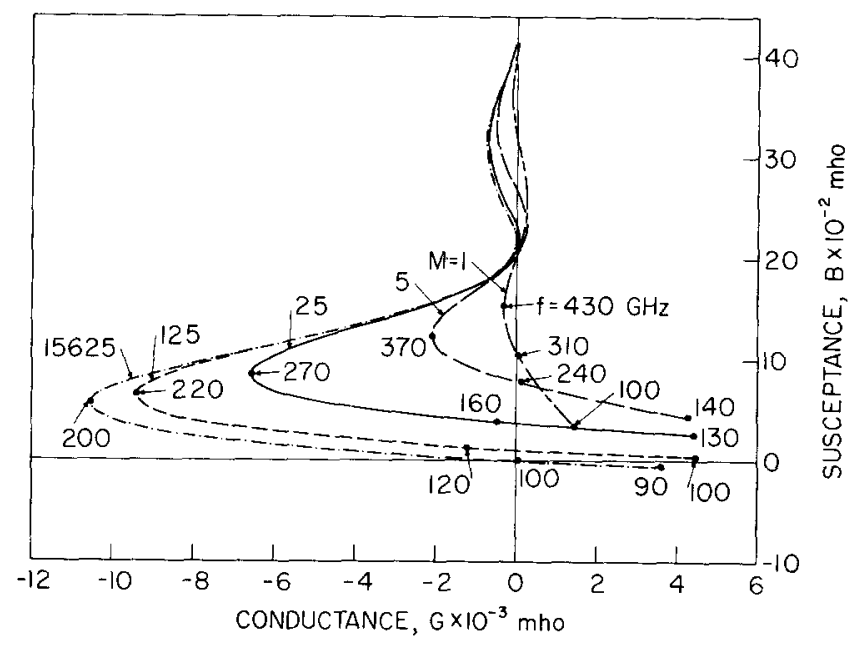

FIG. 8. Small-signal admittance of an IMPATT oscillator with varying $\alpha^{\prime} . \omega_{a}=\omega_{a 0}=2 \pi 100 \mathrm{GHz}, \tau_{\alpha} / \tau=0.1, \delta / W=0.1$,
$\tau_{a} \omega_{a 0}=0.1, A=10^{-10} \mathrm{~m}^{2}$. 
Reducing $M$ while maintaining a constant total current may correspond physically to an increase in electric field, saturated ionization rates, ${ }^{13,15,16}$ and a slight drop in the active region width. This has little effect on the transit phase angle but is sufficient to reduce the multiplication factor $M$ considerably. Assuming that the ionization rate has a saturation trend such that

$$
\alpha_{0}^{\prime}+\frac{1}{M}\left(\frac{\gamma / E_{0}+2}{E_{0} \delta}\right)=\text { constant }
$$

then the negative conductance invariably decreases as the value of $M$ is reduced. This is shown in Fig. 8 . The upward shift of the threshold frequency and the optimum operating frequency are still retained. This suggests that the saturation of the ionization rates which occurs at a very high field and low bias voltage tends to reduce the negative conductance. Hitherto, there have been no well-established data of ionization rates at field strengths above $10^{6} \mathrm{~V} / \mathrm{cm}$ where tunneling is also prevalent. However, such a saturation trend of ionization rates is already discernible in GaAs for a field strength of $5 \times 10^{5} \mathrm{~V} / \mathrm{cm} .^{13}$

\section{ELECTRON-HOLE PAIR-PRODUCTION TIMES AND TUNNELING TIME}

The pair-production relaxation times for electrons and holes in Si have been calculated by Kane. ${ }^{17}$ They are found to increase with decreasing energy. The relaxation time of optical phonon scattering at the energy range of interest is of the order of $6.6 \times 10^{-15}$ sec. $^{17,18}$ Therefore for those energetic electrons and holes which undergo pair-production collisions, the relaxation time cannot, on the average, be much larger than $6.6 \times 10^{-15}$ sec. Otherwise, the energy loss mechanism would be dominated by phonon scattering. The energy loss rate due to optical phonon emissions is greater than that of pair-production ionization when the latter has a relaxation time exceeding

$$
\left(\mathcal{E}_{i} / \mathcal{E}_{r}\right) \times 6.6 \times 10^{-15} \mathrm{sec} \sim 2.64 \times 10^{-13} \mathrm{sec},
$$

where $\mathcal{E}_{i}$ and $\mathcal{E}_{\gamma}$ are energy losses per ionization and per phonon emission, respectively. Therefore, for excitation frequencies not exceeding $10^{12} \mathrm{~Hz}$, the pairproduction ionization can be deemed to be instantaneous.

Tunneling time in a semiconductor was estimated by Keldysh ${ }^{19}$ to be of the order of

$$
\tau_{g}=\left(2 m \mathcal{E}_{g}\right)^{1 / 2} / e E^{1 / 2} \sim 3.4 \times 10^{-14} \mathrm{sec},
$$

where typical values of $\mathscr{E}_{g} \sim 1 \mathrm{eV}$ and $E \sim 10^{6} \mathrm{~V} / \mathrm{cm}$ were used. From another point of view, the tunneling rate expression due to Zener ${ }^{20}$ consists of a tunneling probability term multiplied by Zener oscillation frequency. Therefore it is expected that the tunneling rate will be affected at a frequency comparable to the Zener oscillation frequency which is

$$
\nu_{\mathrm{z}}=e E a / \hbar \sim 1.2 \times 10^{13} \mathrm{~Hz} \text {, }
$$

where $E \sim 10^{6} \mathrm{~V} / \mathrm{cm}$ and $a$ (lattice constant) $\sim 5 \AA$ have been used. Hence, for the frequency range of interest $\left(\leqslant 10^{12} \mathrm{~Hz}\right)$ tunneling can be assumed to be instantaneous.

\section{CONCLUSIONS}

The agreement between the predicted $I-V$ character istics of diodes with both tunneling and avalanche processes and the measured ones indicate the soundness of the formulation developed here. Moreover, the conventional pure tunneling and pure avalanche cases appear as two special cases of the general problem considered here. Of notable interest is the feature that under some conditions a modest amount of tunneling could increase the small-signal negative conductance of an IMPATT oscillator. Operation in the pure tunnel transit-time mode would be attractive for low-noise applications and in particular in high-frequency self oscillating detectors. The formulation is potentially a useful tool for studies of more fundamental problems involving both tunneling and avalanche processes; notably studies of ionization rates and noise at a very high field $\left(\gtrsim 10^{6} \mathrm{~V} / \mathrm{cm}\right)$. The former is especially convenient because the tunneling current and multiplication current appear separated. Due to the lack of experimental data on the ionization rates and drift velocities at the fields of interest, the properties at a somewhat lower field were assumed. Modifications may be needed as new information becomes available. However, it is believed that the essential features given here will be retained.

\section{ACKNOWLEDGMENT}

The authors would like to thank Professor G. Weinreich for a stimulating discussion on tunneling time.

\footnotetext{
*Work supported by the National Aeronautics and Space Administration under Grant No. NGL 23-005-183.

A. G. Chynoweth and K. G. McKay, Phys. Rev. 106, 418 (1957)

${ }^{2}$ W. T. Read, Beil Syst. Tech. J. 37, 401 (1958)

${ }^{3}$ V. K. Aladinskii, Sov. Phys. Semicond. 2, 517 (1968).

${ }^{4} \mathrm{~A}$. Semichon and J. Michel, Proceedings of the Eighth International Conference on Microwaves and Optical Generation and Amplification (Kluwer-Deventer, Amsterdam, 1970).

Amsterdam, 1970).
${ }^{5}$ E. O. Kane, J. Phys. Chem. Solids 12, 181 (1960)

${ }^{5}$ E. O. Kane, J. Phys. Chem. Solids 12, 181 (1960).
${ }^{6}$ C. B. Duke, Tunneling in Solids (Academic, New York, 1969), pp. 40, 41.

${ }^{7}$ C. B. Duke, Tunneling in Solids (Academ

${ }^{8}$ A. G. Chynoweth, W. L. Feldman, C. A. Lee, R. A. Logan, and G.L.

${ }^{8}$ A. G. Chynoweth, W. L. Feldman, C. A
Pearson, Phys. Rev. 118, 425 (1960).

${ }^{9}$ H. Mizumo, Jap. Jap. J. Appl. Phys. 5, 1008 (1966)

${ }^{10}$ P. J. Price and J. M. Radcliffe, IBM J. Res. Dev. 3, 364 (1959).

"P. T. Greiling and G. I. Haddad, IEEE Trans. Microwave Theory Tech.

18, 842 (1970).

${ }^{22}$ M. Singh Tyagi, Solid-State Electron. 11, 99 (1968).

${ }^{13}$ S. M. Sze and G. Gibbons, Appl. Phys. Lett. 8, 111 (1966).

${ }^{14} \mathrm{M}$. Gilden and M. E. Hines, IEEE Trans. Electron Devices 13, 169 (1966).

${ }^{15}$ P. A. Wolff, Phys. Rev. 95, 1415 (1954).

${ }^{16}$ K. G. McKay, Phys. Rev. 94, 877 (1954).

${ }^{17}$ E. O. Kane, Phys. Rev. 159, 624 (1967)

${ }^{18}$ E.O. Kane, J Phys. Soc. Jap. Suppl. 37, 21 (1966)

${ }^{19}$ L. V. Keldysh, Sov. Phys. JETP 20, 1307 (1965)

${ }^{20} \mathrm{C}$. Zener, Proc. Roy. Soc. Lond. A 145, 523 (1934)
} 